\title{
Reduced timing Sensitivity in all-optical switching using flat-top control pulses obtained by the optical fourier transform technique
}

Oxenløwe, Leif Katsuo; Galili, Michael; Mulvad, Hans Christian Hansen; Clausen, A. T.; Jeppesen, Palle

Published in:

International Conference on Photonics in Switching, 2006. PS '06.

Link to article, DOI:

10.1109/PS.2006.4350158

Publication date:

2006

Document Version

Publisher's PDF, also known as Version of record

Link back to DTU Orbit

Citation (APA):

Oxenløwe, L. K., Galili, M., Mulvad, H. C. H., Clausen, A. T., \& Jeppesen, P. (2006). Reduced timing Sensitivity in all-optical switching using flat-top control pulses obtained by the optical fourier transform technique. In International Conference on Photonics in Switching, 2006. PS '06. (pp. 1-3). IEEE. https://doi.org/10.1109/PS.2006.4350158

\section{General rights}

Copyright and moral rights for the publications made accessible in the public portal are retained by the authors and/or other copyright owners and it is a condition of accessing publications that users recognise and abide by the legal requirements associated with these rights.

- Users may download and print one copy of any publication from the public portal for the purpose of private study or research.

- You may not further distribute the material or use it for any profit-making activity or commercial gain

- You may freely distribute the URL identifying the publication in the public portal 


\title{
Reduced timing sensitivity in all-optical switching using flat-top control pulses obtained by the optical Fourier transform technique
}

\author{
L.K. Oxenløwe, M. Galili, H.C. Mulvad Hansen, A.T. Clausen and P. Jeppesen \\ COM•DTU Department of Communications, Optics and Materials, Technical University of Denmark, building \\ 345V, DK-2800, lo@com.dtu.dk
}

\begin{abstract}
For high-speed serial data, timing tolerance is crucial for switching and regeneration. We propose a novel scheme to generate flat-top pulses, for use as gating control pulses. The scheme relies on spectral shaping by a square-shaped filter, followed by a linear transformation of the spectral shape into the time domain, referred to as the optical Fourier transform technique. A 3 ps flat-top pulse derived from a $3 \mathrm{~nm}$ wide square filter is obtained, and used to gate an all-optical OTDM demultiplexer, yielding an error-free timing jitter tolerance of 3 ps for 80 $\mathrm{Gb} / \mathrm{s}$ and $160 \mathrm{~Gb} / \mathrm{s}$ data signals.
\end{abstract}

Keywords: Optical Fourier transform, square pulse, timing jitter, all-optical switching.

\section{Introduction}

High-speed serial data communications is presently regaining a position as a focus area in the optical communications community. An example of this trend is the strong efforts put in 100 G ETDM and Ethernet research worldwide [1]. High-speed serial data may be particularly beneficial for e.g. metro networks or for serial connections between super-computers. Due to speed limitations of electronics, signal processing at high line rates (>100 Gb/s) is currently only possible by optical means. This suggests that e.g. regeneration and add/drop multiplexing will be preferable to perform all-optically in a very fast optical switch, i.e. a switch that is gated by an optical control signal. At high bit rates, timing jitter becomes an important detrimental factor [2], and hence means to introduce jitter tolerance in switches are very attractive. This may be obtained by using a flat-top optical gating pulse, thus creating a flat-top switching window, which will by nature be tolerant to timing fluctuations between gating pulses and data pulses. Previously, a highly bi-refringent fibre has been used to generate a pulse with an elongated top, which was implemented in a $160 \mathrm{~Gb} / \mathrm{s}$ regenerator [3]. Another approach has been to design a sinc-shaped filter corresponding to a squareshaped pulse, which was demonstrated in an $80 \mathrm{~Gb} / \mathrm{s}$ demultiplexing experiment [4].

In this paper, we show a novel approach to generate square switching windows and demonstrate its use in a 80 and $160 \mathrm{~Gb} / \mathrm{s}$ OTDM demultiplexing experiment. The technique relies on the optical Fourier transform technique, as e.g. described in [5], where a certain spectral shape is transformed to the same shape temporally by an appropriate combination of frequency chirping and dispersion. We show that the demultiplexer obtains a 3 ps timing jitter tolerance this way, as opposed to $1 \mathrm{ps}$ when a Gaussian control pulse is used.

\section{Principle and experimental set-up}

\subsection{Principle: The optical Fourier transform technique}

Figure 1 shows a sketch of the principle. The basic idea is to let a Fourier transform pair swap domains by a linear transformation, by adding a linear chirp to the pulse and send it through a dispersive medium [5].

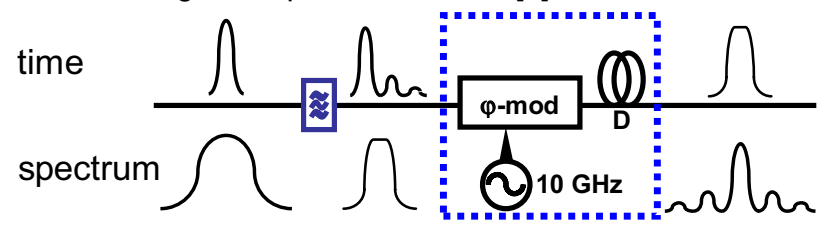

Figure 1 : Principal sketch of square pulse generation.

A temporally narrow, and hence spectrally wide, pulse is injected into a filter with a square-like filter function. The output of the filter will thus be spectrally square, and correspondingly temporally sinc-function-like. Using a sinusoidal phase modulation, a linear chirp is added. By carefully balancing the added chirp and the dispersion of a length of fibre, the transform pairs will swap their domain shapes. In our case we turn a square spectrum into a temporal square pulse.

\subsection{Experimental set-up}

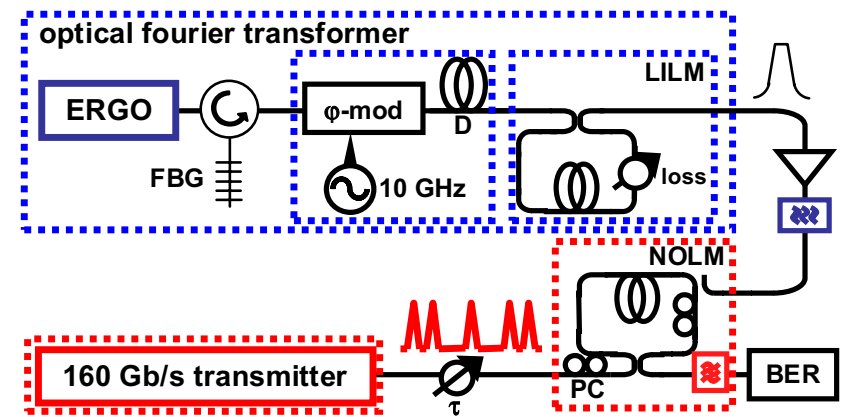

Figure 2 : Experimental set-up for square pulse generation and subsequent switching characterisation.

The main goal in this paper is to generate a square switching window, to reduce the timing jitter influence on the switching performance. To this end, we construct a flat-top square-shaped pulse, which we use as control pulse in an ultra-fast fibre-based switch - here, a non- 
linear optical loop mirror (NOLM) - which has a switching window determined by the control pulse shape.

Referring to figure 2, a 2 ps pulse with a $3.5 \mathrm{~nm} 3-\mathrm{dB}$ broad spectrum centred at $1545 \mathrm{~nm}$ (derived from an Erbium glass oscillating pulse source, ERGO) is filtered in a specially designed fibre Bragg grating (FBG) with a square filter function with a 3-dB bandwidth of $2 \mathrm{~nm}$. This square spectrum is injected into a chirp-dispersion unit, where it is phase modulated ( $\varphi$-mod) at $10 \mathrm{GHz}$. By matching the induced chirp to the total dispersion (D) of a dispersive medium (here $4.5 \mathrm{~km}$ standard single mode fibre SSMF), the transformation from the spectral domain to the temporal domain is complete, and square pulses are obtained. These pulses have some pedestals, which are suppressed by passing them through a loss-imbalanced loop mirror (LILM), which works by virtue of a differential self-phase shift in the two loss-imbalanced arms. The LILM contains $500 \mathrm{~m}$ HNLF. The square pulses are subsequently amplified and used to gate a NOLM demultiplexer, which demultiplexes an 80 or $160 \mathrm{Gbit} / \mathrm{s}, 2^{7}$ 1 PRBS, single-polarisation optical time division multiplexed (OTDM) data signal down to $10 \mathrm{~Gb} / \mathrm{s}$ for subsequent bit error rate measurements. The temporal displacement between data and control pulses is controlled by an optical time delay $(\tau)$.

\section{Flat-top pulse generation and characteristics}
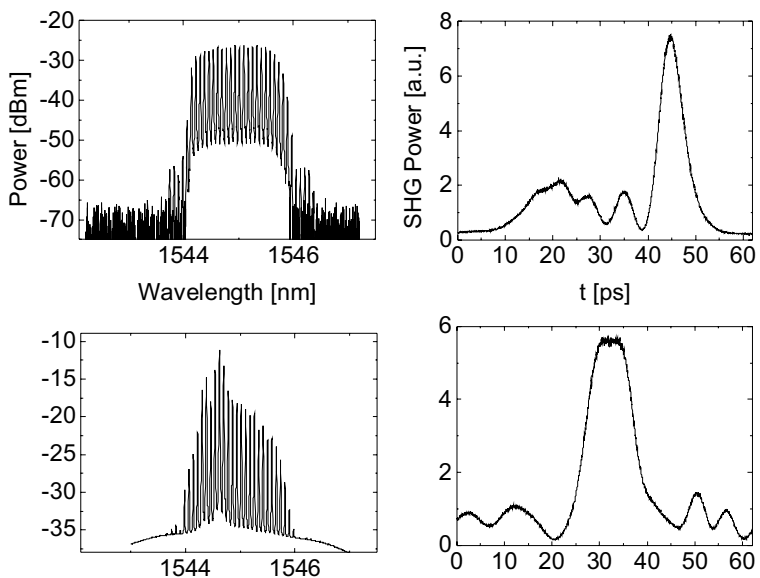

$\mathrm{t}[\mathrm{ps}]$

Wavelength [nm]
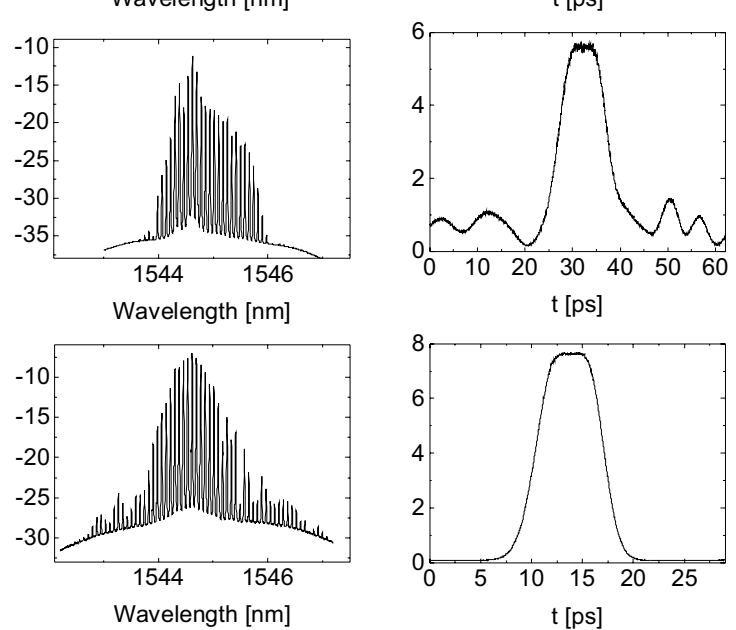

Figure 3 : Experimental results for flat-top pulses. Top: output of FBG (left: spectrum, right: pulse shape). Middle: output of chirp-dispersion unit. Bottom: output of LILM.

Figure 3 shows results on the generation of the square pulses in terms of spectra and temporal cross-correlations with a 1.7 ps pulse. A 2 ps Gaussian pulse with a $3.5 \mathrm{~nm}$ Gaussian spectrum is injected into a fibre Bragg grating filter. The output of the FBG is shown in figure 3 (top) in terms of spectral and temporal shapes. As seen, the spectrum is a square with a $3-\mathrm{dB}$ bandwidth of about 2 $\mathrm{nm}$. The corresponding pulse is sinc-like with a central peak and a "ringing" tail of trailing pulses covering about $40 \mathrm{ps}$ in total.

Figure 3 (middle) shows the output of the chirp-dispersion unit. The phase modulator runs at $10 \mathrm{GHz}$ with a $1.5 \pi$ shift from peak to peak. At the output of the dispersive medium, the added chirp is compensated so that the square spectral shape has now been transferred onto the temporal shape, yielding a square pulse (figure 3 (middle, right)) with a 4 ps flat top and an 11 ps FWHM. There are some residual pulses due to imperfectly compensated chirp, which are suppressed by the LILM. The output of the LILM is shown in figure 3 (bottom), and a clear square pulse is obtained. There is an $18 \mathrm{~dB}$ extinction ratio on the square pulse, and it has a 3 ps wide flat top. Its FWHM is $\sim 6.5$ ps (cross-correlated with the 1.7 ps sampling pulse, so in reality the FWHM is narrower) and its spectral envelope (now appearing more sinc-like) has a $3-\mathrm{dB}$ bandwidth of $\sim 1 \mathrm{~nm}$ and a $20-\mathrm{db}$ bandwidth of $\sim 4 \mathrm{~nm}$.

\section{Switching performance - system validation}

In order to demonstrate the use of the generated square pulse in a system, it is used as control pulse in an OTDM NOLM-demultiplexer. The OTDM data is generated by the use of a second ERGO pulse source running at $10 \mathrm{GHz}$, data modulated with a $2^{7}-1$ PRBS sequence in a $\mathrm{LiNbO}_{3}$ modulator before being multiplexed to 80 or $160 \mathrm{~Gb} / \mathrm{s}$ in a polarisation and PRBS maintaining passive fibre-delay OTDM multiplexer. The data signal is at $1557 \mathrm{~nm}$ and the NOLM (as the LILM) uses a $500 \mathrm{~m}$ highly non-linear fibre with zero dispersion at $1551 \mathrm{~nm}$ and a dispersion slope of $0.017 \mathrm{ps} / \mathrm{nm}^{2} / \mathrm{km}$, so there is very low walk-off between control and data, and very low pulse broadening. This assures the switching window shape to be determined by the control pulse shape.

\section{$4.180 \mathrm{~Gb} / \mathrm{s}$ characterisation}

Figure 4 shows the BER performance for demultiplexing an $80 \mathrm{~Gb} / \mathrm{s}$ OTDM signal. The operation is successful and error-free performance is obtained. The BER curve shows no sign of an error floor, and the demultiplexed eye is clear and open (figure 4 , bottom right corner). There is an only 2 $\mathrm{dB}$ penalty with respect to the $10 \mathrm{~Gb} / \mathrm{s}$ back-to-back (b-b). Figure 4 (top right) shows the BER performance of all the 8 multiplexed channels in terms of their receiver sensitivity, i.e. the minimum receiver power to obtain an error-free $\operatorname{BER}\left(10^{-9}\right)$, when the square control pulse is set on one channel after the other. All 8 channels are error free with very similar sensitivities $(\sim-35 \mathrm{dBm}$ with a spread of 0.5 $\mathrm{dB})$. 


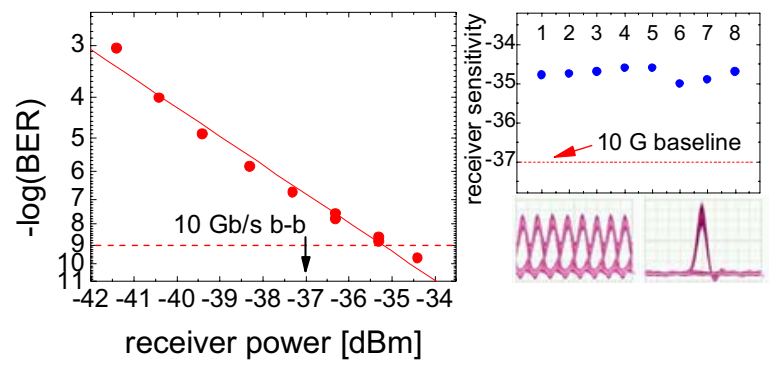

Figure 4 : Experimental results for demultiplexing $80 \mathrm{~Gb} / \mathrm{s}$.

In order to evaluate the influence of the flat top of the control pulse, it is stepped across a data channel and at each step, the BER is evaluated - the receiver power is set to the receiver sensitivity plus $5 \mathrm{~dB}$ more for this measurement. This reveals how big a time displacement between data and control can be tolerated, and is a static measure of how much timing jitter can be tolerated by this switch configuration. The result is shown in figure 5 .

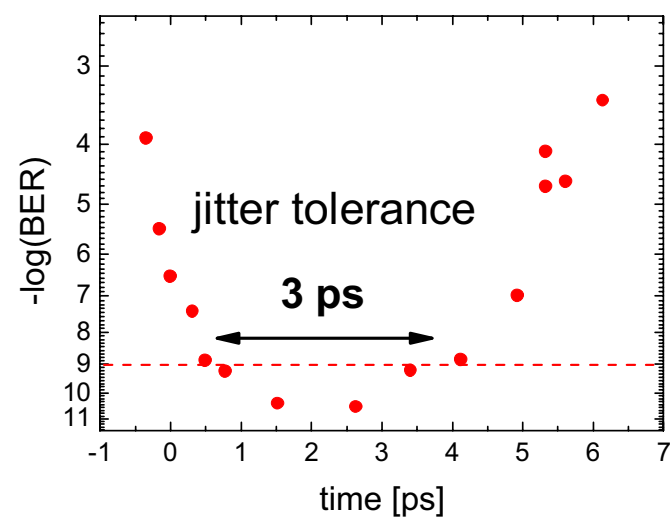

Figure 5 : BER over control-data displacements $-80 \mathrm{~Gb} / \mathrm{s}$.

As seen from figure 5 , the result is strikingly clear: Within the 3 ps flat top, the demultiplexing remains error-free. 80 $\mathrm{Gb} / \mathrm{s}$ data has a time slot of $12.5 \mathrm{ps}$, so the control pulse is narrower than needed for this bit rate. Still, with the narrow data pulses used here, the conclusion is clear - the jitter tolerance is given by the flat top.

\section{$2.2160 \mathrm{~Gb} / \mathrm{s}$ characterisation}

With a 3 ps flat top and a FWHM $~ 6.5$ ps, the pulse should also work for $160 \mathrm{~Gb} / \mathrm{s}$ switching, though it is of concern whether the wide FWHM would cause neighbouring channels to be switched through, creating inter-symbol interference (ISI). As seen from figure 6, though, the performance is error-free. In fact, all 16 channels are error-free and the average sensitivity is -34.5 $\mathrm{dBm}$, i.e. only $0.5 \mathrm{~dB}$ worse than for $80 \mathrm{~Gb} / \mathrm{s}$.

Figure 6 shows the timing tolerance for the $160 \mathrm{~Gb} / \mathrm{s}$ demultiplexing case. For comparison, the demultiplexing is also performed with a Gaussian 2 ps wide pulse. Again, the receiver power is set to $5 \mathrm{~dB}$ above the receiver sensitivity for both pulse types to allow for some margin.

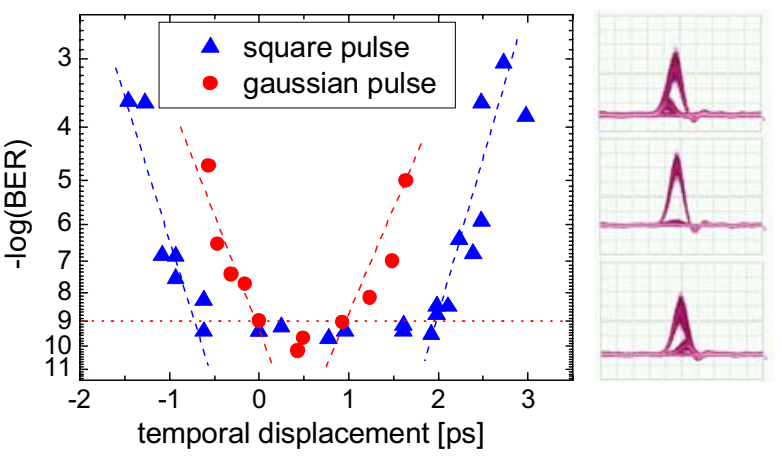

Figure 6 : BER over control-data displacements $-160 \mathrm{~Gb} / \mathrm{s}$.

The square pulse is seen to maintain error-free performance with a 2.6 ps tolerance to temporal displacement, in contrast to the 3 ps at $80 \mathrm{~Gb} / \mathrm{s}$. The Gaussian pulse on the other hand, only ensures error-free operation within a 1 ps tolerance. So the improvement is evident. The reason for the reduction of tolerance with respect to the $80 \mathrm{~Gb} / \mathrm{s}$ case, is that the FWHM of the square pulse is just a bit too wide. This results in neighbouring channels being switched through sooner that desired, when a time displacement is introduced, leading to ISI. This explains the steeper BER curves in figure 6 when using the square pulse, and is also demonstrated by the eye diagrams to the right in figure 6 - here neighbouring channels are clearly seen to creep in at displacements to either side. Since at $80 \mathrm{~Gb} / \mathrm{s}$ there are no immediate neighbours (with 2 ps data pulses), this steep rise is not observed.

\section{Conclusion}

We have described a novel concept for generating jitter tolerant control signals for high-speed switching. By virtue of the optical Fourier transform technique, a squareshaped spectrum was transformed into a square-shaped pulse. This was used to demultiplex from 80 and 160 to 10 $\mathrm{Gb} / \mathrm{s}$ with increased jitter tolerance from 1 to 3 and $2.6 \mathrm{ps}$, respectively.

\section{Aknowledgments}

OFS Fitel Denmark for providing HNLFs. The Danish Research Council for funding through the project Ultra-Net.

\section{References}

[1] Various authors, OFC'06 postdeadline papers session IV (e.g.http://www. ofcnfoec.org/conference program/Accept ed Postdeadline Papers.aspx.), 2006

[2] D. Zibar et al, OFC'06, paper OTuB2, 2006

[3] S. Watanabe et al, OFC'03, paper PD 16-1, 2003

[4] J.H. Lee et al, J LT, 21(11) (2003), pp 2518

[5] M. Nakazawa et, PTL, 16(4) (2004), pp 105 